\title{
La Incidencia del Contexto Interno Docente en la Aparición del Síndrome del Quemado por el Trabajo en Profesionales de la Enseñanza
}

\section{Incidence of the Teaching Internal Context in the Emergence of Burnout in Teaching Profession}

\author{
Jordi Longas \\ Universidad Ramon Llull
}

\author{
Andrés Chamarro \\ Universidad Autónoma de Barcelona
}

\author{
Jordi Riera \\ Universidad Ramon Llull
}

\author{
Ramón Cladellas
}

\begin{abstract}
Resumen. La investigación tiene como objetivo profundizar en el estudio de la relación entre el síndrome de quemarse por el trabajo en la docencia y los factores organización escolar y gestión de aula. La muestra está formada por 1.360 docentes de Cataluña (España), a los que se ha aplicado el MBI, un cuestionario que recoge datos sociodemográficos y profesionales, y el cuestionario de factores del contexto interno docente, diseñado y validado específicamente para esta investigación. Los resultados muestran índices de riesgo psicosocial en los docentes y confirman que: a) las escuelas próximas al paradigma de la escuela que aprende son más saludables y b) la organización escolar y la gestión de aula son factores preventivos fundamentales ante los riesgos psicosociales en la docencia. Se concluye que el modelo de organización escolar denominado escuela que aprende pudiera anticiparse como el patrón de cultura profesional y organizativa propio de las escuelas más saludables.

Palabras clave: Síndrome de Quemarse por el Trabajo, docentes, organización escolar, gestión de aula, escuela saludable.
\end{abstract}

\begin{abstract}
The aim of this study is analyse the relation between the teacher's burnout and the school organisation and classroom management. The sample is formed by 1.360 teachers of Catalonia (Spain), to which has applied the MBI, a questionnaire that collects social and professional data, and the questionnaire of Factors of the Teachers Internal Context, designed and validated specifically for this investigation. Results show indexes of psychosocial risk in the educational and confirm that: a) the next schools to the paradigm of the learning organization are healthier, and b) the school organisation and classroom management are fundamental preventive factors in front of the psychosocial risks in the teaching. We conclude that the model of school organization called learning school could be the pattern of healthier organizational culture at schools.

Keywords: Burnout, teachers, school organization, classroom management, healthy school.
\end{abstract}

En la última década, se ha desarrollado un gran interés por el estudio del estrés, los riesgos psicosociales relacionados con el trabajo y su repercusión sobre la salud de los trabajadores (Cladellas y Badia, 2010; Cladellas y Castelló, 2011; Extremera, Duran y Rey, 2010; Gil-Monte, 2007). Entre las enfermedades asociadas al estrés laboral, el síndrome de burnout o síndrome de quemarse por el trabajo (SQT), como propone Gil-Monte (2003), es cada vez más conocido y lamentablemente más extendido. Aunque no exclusivamente, suele afectar a personas incluidas en las denominadas profesiones de ayuda (salud, educación, servicios sociales, etc.), cuyas tareas laborales tienen un componente central de ayuda a los demás y de gran intercambio emocional. El SQT se describe como una respuesta personal a situaciones de inadecuación pro-

La correspondencia sobre este artículo deberá ser dirigida a Andrés Chamarro, Universidad Autónoma de Barcelona, Edificio B, Campus de Bellaterra, 08193 Bellaterra. E-mail: Andres.chamarro@uab.es fesional frente a demandas generadas en el trabajo. Se considera un fenómeno tridimensional que integra: a) sentimientos de agotamiento emocional; b) actitudes de despersonalización (cinismo, distanciamiento, frialdad...) hacia alumnos, padres y colegas; y c) baja realización personal, generalmente asociada a la disminución de la eficacia en el trabajo docente (Maslach, 1999; Maslach, Schaufeli y Leiter, 2001). No obstante, hay una creciente tendencia a considerar el agotamiento emocional como la dimensión central del SQT (GilMonte, 2007; Halbesleben y Demerouti, 2005; Melamed, Shiron, Toker, Berliner y Shapira, 2006) y a valorar la aparición de sentimientos de culpa como instigador de un círculo vicioso en el proceso de desarrollo del SQT (Gil-Monte, 2005).

Los riesgos psicosociales han emergido en las últimas décadas como uno de los principales retos para la salud laboral. El 22-30\% de los trabajadores de la Unión Europea está afectado por estrés laboral (Mansilla, 2008), asociándose al 55\% de las bajas 
laborales (Agencia Europea para la Seguridad y la Salud en el Trabajo, 2009) y comportando un costo económico superior a los 20.000 millones de euros para los países miembros de la U.E. (Gascón, Olmedo y Ciccotelli, 2003). La prevalencia del SQT en profesionales de la enseñanza varía según los contextos e investigaciones, pero siempre presenta niveles importantes (Vercambre, Brosselin, Gilbert, Nerrière y Kovess, 2009). Algunos estudios específicos con docentes españoles indican que la afectación del SQT oscila entre el $48.9 \%$ (Ayuso y Guillén, 2008) y el $25.7 \%$ (Otero-López, Castro, Villardefrancos y Santiago, 2009), de los cuales entre el 3-4\% presentarían una sintomatología grave (Manassero et al., 2000). El análisis por dimensiones varía entre 26.4$21.5 \%$ con alto agotamiento emocional, $6.6-4 \%$ con alta despersonalización, y 33-7.3\% con baja realización personal, según Durán, Extremera y Rey (2001) y Extremera, Durán y Rey (2010), ésta última siguiendo criterios más restrictivos para la valoración de los niveles de afectación. En estudios realizados en países culturalmente próximos, Unda, Sandoval y Gil-Monte (2008) concluyeron que la prevalencia de burnout en una muestra de profesores mejicanos era del $17 \%$. Figueiredo-Ferraz, Gil-Monte y Grau (2009), encontraron que un $14.20 \%$ de 211 profesores portugueses eran identificados en el perfil 1, estaban afectados por el SQT, pero no presentaban altos niveles de culpa, y sólo un $1.9 \%$ en el perfil 2, estaban afectados por el síndrome y presentaban altos sentimientos de culpa. De la misma manera, Gil-Monte, Carlotto y Gonçalves (2011) estimaron que el $12 \%$ de los profesores brasileños se identificaban con el perfil 1 y sólo el $5.6 \%$ de la muestra se ajustaba al perfil 2 .

Las cifras, más allá de la sobredimensión y el alarmismo con el que es tratado el fenómeno por los medios de comunicación (Botella, Longás y Gómez, 2007), nos acercan a los importantes costes humanos y laborales del SQT en la enseñanza y la amenaza que supone para la calidad educativa (Arís, 2009; Moriana y Herruzo, 2004).

Se considera que el SQT aparece como resultado de una situación crónica de estrés negativo, generado progresivamente por la interacción negativa entre las características personales y las características del entorno laboral (Gil-Monte, 2005; Maslach, et al., 2001). La literatura identifica dentro del contexto escolar diversas variables relacionadas con el SQT. Algunas remarcables son: el compromiso del profesorado (Durán, Extremera, Montalbán y Rey, 2005; Salanova, Schaufeli, Llorens, Peiró y Grau, 2000); los problemas del aula y el desinterés de los alumnos (Manassero, Fornés, Fernández, Vázquez y Ferrer, 1996; Otero-López et al., 2009); las deficientes condiciones laborales (Peiró, 1992); el clima escolar (Peiró y Salvador, 1993); los horarios extremos de los docentes (Cladellas y Castelló, 2011); o las elevadas demandas sobre los docentes (Vercambre et al., 2009).
En cuanto a su manifestación, desde el punto de vista del género, los hombres parecen mostrar valores superiores en despersonalización y las mujeres tienden a mostrar mayor agotamiento emocional (Ayuso y Guillén, 2008; Llorens, García y Salanova, 2005; Moriana y Herruzo, 2004). Respecto a la edad, los resultados son controvertidos, aunque tienden a apoyar que el agotamiento emocional se incrementa con la edad y los años de profesión (Marqués, Lima y Lópes, 2005) situándose las etapas más críticas entre los 43-57 años (Llorens et al., 2005) y los 40-49 años (OteroLópez et al., 2009). También el nivel educativo impartido ha mostrado relación con el SQT, aumentando a medida que asciende el ciclo educativo, siendo los profesores de secundaria los más afectados (Otero-López et al., 2009), sobre todo en cuanto a la despersonalización y a la realización personal. Por el contrario, la ausencia de dificultades para gestionar la clase parecen determinantes a la hora de evitar la aparición del SQT (Pillay, Goodard y Wilss, 2005).

Hay coincidencia en considerar el SQT como la expresión del desajuste entre la persona y la organización, provocado por el conflicto o la disonancia entre los valores de la organización y los personales (Villanueva et al., 2008). No obstante, la mayoría de investigaciones se centran en la perspectiva individual (características demográficas, profesionales, cognitivas y afectivas) que modulan el estrés percibido (Extremera et al., 2010; Gil-Monte, 2005; Martínez, Grau, Llorens, Cifré y García-Renedo, 2005). En general, los estudios del contexto docente reconocen la influencia del entorno (cambios normativos, relaciones agresivas con familias y alumnos, sobrecargas laborales, pérdida de prestigio, etc.), de la propia organización de los centros educativos (burocratización, falta de apoyo institucional, política y estructura institucional, etc.) y de los sistemas de relación interpersonal (conflicto y ambigüedad de rol, escaso apoyo social, incertidumbre en la relación con alumnos y familias, falta de reciprocidad, contagio, etc.) en el proceso de desarrollo del SQT. Pese a ello, existe menos investigación alrededor de modelos concretos de organización escolar (Ahghar, 2008; Boada, de Diego y Agulló, 2004).

En este sentido, el modelo de escuela que aprende (Bolívar, 2001; Gairín, 1999, 2000; Santos Guerra, 2000) pudiera anticiparse como el patrón de cultura profesional y organizativa propio de las escuelas más saludables al caracterizar, según los avances en teoría de dirección, a las mejores organizaciones (estrategia organizativa, relación con el entorno, innovación y evaluación, gestión del conocimiento, aprendizaje colaborativo, etc.); y responder a planteamientos pedagógicos críticos (Tardif, 2004) en el desarrollo de la profesionalidad docente (proyecto compartido, orientación hacia el alumno, acción cooperativa, etc.). En consonancia con este modelo de escuela, cabe analizar qué sucede en el aula (Gairín, 1999), lugar en el 
Tabla 1. Distribución de los centros y docentes de la muestra

\begin{tabular}{|c|c|c|c|c|c|c|}
\hline & Población centros & Muestra & $\%$ tot & Población docentes & Muestra & $\%$ tot \\
\hline Cataluña & 410 & 38 & 9.26 & 15.170 & 1360 & 8.96 \\
\hline Barcelona & 129 & 15 & 11.6 & 4.773 & 532 & 11.14 \\
\hline$>100.000$ hab & 120 & 9 & 7.5 & 4.440 & 372 & 8.38 \\
\hline$<100.000$ hab & 161 & 14 & 8.7 & 5.957 & 456 & 7.66 \\
\hline
\end{tabular}

que se expresa el estilo docente y las competencias para enseñar. Para estos autores, la preocupación por el bienestar emocional de los alumnos y el liderazgo efectivo de las personas, los grupos y la programación incide en el clima positivo del aula y reduciría la aparición del SQT. En este sentido, consideramos que los centros concertados promueven un ejercicio de la profesión docente más saludable ante la aparición del SQT en tanto que su organización busca anticiparse y adaptar la tarea docente a los cambios del entorno, siendo activos en la incorporación de las demandas del entorno y la flexibilidad de su propia organización.

De acuerdo con lo argumentado, consideramos necesario analizar las relaciones entre el contexto interno docente -aula y escuela- y el SQT, buscando identificar las características de organización docente que permiten prevenir el SQT de acuerdo a un modelo de escuela y de docencia más saludable. Desde este punto de vista, el estudio tiene un doble objetivo: a) analizar la prevalencia del SQT en el profesorado de centros concertados y b) describir el contexto docente y determinar su relación con el SQT. A este respecto, se formulan dos hipótesis: 1) que la prevalencia del SQT en los centros concertados es inferior a la de otros estudios, y 2) que los factores del contexto interno docente son mejores predictores del SQT que las variables sociodemográficas y de historia profesional.

\section{Método}

\section{Participantes}

Participaron un total de 1.360 docentes de enseñanza infantil, primaria y secundaria pertenecientes a 38 centros concertados de Cataluña (España), con una edad media de 39.35 años $(S D=9.97)$. Un $69 \%$ eran mujeres. Para seleccionar la muestra se hizo una primera selección de 60 centros concertados según su distribución en función del número de habitantes de la ciudad en la que trabajan: a) ciudad de Barcelona, b) ciudades catalanas de más de 100.000 habitantes, c) ciudades o pueblos de Cataluña de menos de 100.000 habitantes. En esta selección no se consideró el nivel educativo como una variable a considerar porque casi la totalidad de centros concertados de esta agrupación son centros integrados que imparten las diferentes eta- pas en un mismo centro. Los 1.360 cuestionarios corresponden a 38 centros que representan el $9.26 \%$ del total de centros de la población. La composición de la muestra ha sido la siguiente: 15 centros y 532 docentes de la ciudad de Barcelona; 9 centros y 372 docentes de Cornellà, Granollers, Hospitalet de Llobregat, Lleida, Mataró, Olot, Reus, Sabadell y Tarrasa (ciudades de más de 100.000 habitantes); y 14 centros y 456 docentes de Centelles, Gavà, La Garriga, Malgrat de Mar, Olesa de Montserrat, Sant Feliu de Llobregat, Sant Sadurní d'Anoia, Sant Vicenç dels Horts, Torrelles de Llobregat, Valldoreix y Vilanova i la Geltrú (ciudades o pueblos de menos de 100.000 habitantes).

La Tabla 2 recoge las principales características de la muestra de docentes en función del género y la edad, así como de las variables socioprofesionales vinculadas al ejercicio docente: años de ejercicio como docente, etapa en la que se imparte la docencia, estar ejerciendo o haber ejercido un cargo directivo o de coordinación, años de experiencia como tutor/a y número de alumnos a los cuales se da clase durante el trimestre.

Tabla 2. Características sociodemográficas y profesionales de la muestra

\begin{tabular}{|c|c|c|c|}
\hline \multicolumn{2}{|c|}{ Variable } & \multirow{2}{*}{$\frac{n}{422}$} & \multirow{2}{*}{$\frac{\%}{31.0}$} \\
\hline Género & Hombre & & \\
\hline & Mujer & 938 & 68.9 \\
\hline \multirow[t]{3}{*}{ Edad } & $<30$ & 317 & 23.3 \\
\hline & $31-50$ & 826 & 60.7 \\
\hline & $>51$ & 217 & 15.9 \\
\hline \multirow[t]{3}{*}{ Años de profesión } & $<10$ & 541 & 39.8 \\
\hline & $11-20$ & 414 & 30.4 \\
\hline & $>21$ & 405 & 29.8 \\
\hline \multirow[t]{3}{*}{ Etapa } & Inf/Prim & 677 & 49.8 \\
\hline & ESO & 476 & 35 \\
\hline & PostObli & 207 & 15.2 \\
\hline \multirow[t]{2}{*}{ Cargo directivo } & Sí & 509 & 38.8 \\
\hline & No & 804 & 61.2 \\
\hline \multirow[t]{5}{*}{ Años de tutoría } & 0 & 209 & 15.5 \\
\hline & $1-5$ & 345 & 25.7 \\
\hline & $6-10$ & 252 & 18.7 \\
\hline & $11-15$ & 148 & 11.0 \\
\hline & $>15$ & 390 & 29.0 \\
\hline \multirow[t]{5}{*}{$\mathrm{N}^{\circ}$ alumnos } & $<50$ & 420 & 31.3 \\
\hline & $50-100$ & 297 & 22.1 \\
\hline & $101-150$ & 281 & 20.9 \\
\hline & $151-200$ & 162 & 12.1 \\
\hline & $>200$ & 183 & 13.6 \\
\hline
\end{tabular}




\section{Instrumentos}

Cuestionario de datos sociodemográficos y profesionales: El cuestionario interroga sobre algunas variables de tipo sociodemográfico y de contexto e historia profesional de los docentes, concretamente: género, edad, años de experiencia docente, etapa educativa, ejercer o haber ejercido algún cargo directivo o de coordinación, años de experiencia como tutor y número de alumnos a los cuales se da clase. Este cuestionario ha sido diseñado específicamente para esta investigación.

Maslach Burnout Inventory (MBI): Permite medir las tres dimensiones del SQT: agotamiento emocional, despersonalización y realización personal (Maslach y Jackson, 1986), en su versión española (Seisdedos, 1997). Está constituido por 22 ítems, divididos en tres subescalas, que en nuestra aplicación se han utilizado en términos de intensidad (Calvete y Villa, 1999). En el actual estudio, la subescala de Agotamiento Emocional (9 ítems) ha mostrado una fiabilidad de .87 . La subescala de Despersonalización (5 ítems) mostró una fiabilidad baja .55, coincidiendo con otros estudios (Gil-Monte y Peiró, 1999). La subescala de Realización Personal (8 ítems) ha mostrado una fiabilidad aceptable de .70 .

Cuestionario de factores del contexto interno docente: El cuestionario fue diseñado específicamente para la realización de esta investigación con el objetivo de evaluar la percepción que tienen los docentes de las características del contexto próximo -escuela y aula- con el que interactúa profesionalmente de modo directo. A partir de una revisión de la literatura (Bolívar, 2001; Gairín, 1999 y 2000; Jennings y Greenberg, 2008; Tardif, 2004) se redactaron 80 ítems para recoger información de: el liderazgo prosocial del aula, la gestión de los procesos de enseñanza aprendizaje, el logro eficaz de los objetivos, la participación de los docentes, la adecuada distribución de funciones y recursos, la existencia de un proyecto educativo sólido y aceptado, la buena comunicación interna, el trabajo en equipo, el compromiso con la acción tutorial y el acompañamiento de los alumnos, la vertebración del currículum mediante una planificación efectiva, la orientación hacia el aprendizaje y la existencia de prácticas de evaluación interna como sistema para alimentar la mejora continua. Una primera administración de este cuestionario a 28 docentes voluntarios permitió un primer ajuste morfosintáctico y de contenido, quedando una primera versión depurada de 68 ítems. Un posterior estudio piloto con 101 docentes de todos los niveles educativos, nos llevó a reducir el cuestionario a la versión de 29 ítems utilizada en el estudio actual. La versión utilizada consta de dos factores. Un primer factor denominado "Organización escolar y gestión pedagógica”, está formado por 18 ítems que evalúan: Sistema de organización y coordinación de la escuela; Estilo de dirección/ liderazgo; Equipo docente; Políticas del centro educativo destinadas a asegurar la atención a los alumnos y la convivencia; Gestión del currículum eficaz y abierta a la mejora continua. La consistencia interna de este factor es de .89. El segundo factor, denominado "Gestión de Aula", está formado por 11 ítems que evalúan: Tipo de relación establecida con los alumnos al aula; Grado de control de los grupos; y Satisfacción en relación al aprendizaje. La consistencia interna de este factor es de .84 .

\section{Procedimiento}

Los centros fueron invitados a participar en la investigación con la condición de obtener cuestionarios de un mínimo del $75 \%$ de los miembros del claustro. El profesorado participante era informado del anonimato de los datos y consintió expresamente su participación. Además de asegurar la confidencialidad, se ofreció explícitamente la posibilidad de desestimar participar en el estudio. Los cuestionarios fueron administrados en reuniones de claustro, en presencia de un miembro del equipo investigador y se respondieron en menos de 20 minutos. Como contrapartida de la colaboración se entregó a cada sujeto la corrección de su MBI y, a cada centro, los resultados globales del MBI y la comparación con el conjunto de la muestra.

\section{Análisis de datos}

Se ha llevado a cabo un análisis descriptivo para las tres dimensiones del MBI, incluyendo el cálculo de porcentajes de docentes con niveles bajo, medio o alto según su distribución por percentiles (Seisdedos, 1997). Se ha desestimado considerar con SQT a aquellos docentes que puntúan en niveles altos en una sola dimensión y hemos adoptado el criterio más restrictivo de Manassero et al. (1996), según el cual sólo los docentes con puntuaciones elevadas en 2-3 dimensiones serían indicativos de SQT. También se ha realizado un análisis descriptivo de los factores del contexto interno docente. Para las dimensiones del MBI y los factores del contexto docente se han realizado sucesivos análisis de varianza unifactoriales para las variables socioprofesionales. Finalmente, para comprobar el grado en que las dimensiones del contexto interno docente predicen el SQT, pero controlando el efecto de los aspectos sociodemográficos y profesionales, se ha realizado un análisis de regresión jerárquica sobre las dimensiones del MBI en las que las variables socioprofesionales han sido introducidas en primer lugar, la Organización Escolar en segundo término y finalmente la Gestión Aula. 


\section{Resultados}

\section{Niveles de SQT}

Los valores que indican el nivel del SQT según el MBI en la muestra estudiada se presentan en la Tabla 3. Atendiendo los valores de corte fijados para la corrección del MBI en orden a los niveles de riesgo alto, moderado y bajo, se observa que: el $14.2 \%$ de los docentes de la muestra tienen niveles de riesgo alto de agotamiento emocional; el $9.9 \%$ de docentes de la muestra presenta alta despersonalización; y el $10.1 \%$ de los docentes de la muestra tienen bajos niveles de realización personal, vinculada a la autopercepción de baja eficacia profesional, y por lo tanto en situación de riesgo.

El análisis más detallado por dimensiones (ver Tabla 4) nos indica que el $20.1 \%$ de los sujetos de la muestra se encuentra en niveles de riesgo alto en una sola dimensión, el $5.1 \%$ de la muestra tiene riesgo alto en 2 dimensiones y $1.3 \%$ de los sujetos de la muestra presentan niveles de riesgo alto en las tres dimensiones del MBI. De acuerdo con estos criterios utilizados, los docentes de la muestra apuntan hacia una situación de riesgo en agotamiento emocional y despersonalización, y resultados de menor riesgo en realización personal. Así, 73.4\% de los docentes no presenta riesgo, mientras que el $26.6 \%$ de los docentes manifiestan diferentes grados de SQT, si bien sólo un $6.4 \%$ de la muestra se encuentra en situación de riesgo muy alto (valores elevados en 2 y 3 dimensiones en riesgo). Englobados en este último grupo, el $1.3 \%$ de los docentes que han obtenido puntuaciones elevadas en las tres dimensiones y representan el porcentaje de docentes que se encuentran en una situación de riesgo severo.

En relación al agotamiento emocional (ver Tabla 4) no se observan diferencias de género, pero sí un aumento en función de la edad $(p<.001)$, de los años de ejercicio profesional $(p<.001)$ y de los años de tutoría $(p<.001)$. Respecto a la despersonalización (ver Tabla 4) los hombres presentan puntuaciones significativamente más elevadas que las mujeres $(p<$ .001). También se aprecian diferencias en función de la edad $(p<.05)$ y de la etapa en la que se ejerce la docencia $(p<.001)$. Los docentes de ESO y Bachillerato presentan valores más elevados en esta dimensión. Finalmente, el número de alumnos a los cuales se imparte clase también muestra diferencias significati- vas, siendo los docentes que tienen menos de 50 alumnos a su cargo los que tienen significativamente menores valores de despersonalización $(p<.001)$. Los valores de realización personal (ver Tabla 4) muestran diferencias significativas en función de la edad $(p<.05)$, los años de profesión $(p<.001)$ y la etapa educativa $(p<.001)$.

\section{Factores del contexto interno docente}

Los estadísticos descriptivos para el contexto interno del docente (Organización Escolar/ Gestión Pedagógica y Gestión Aula) se muestran en la Tabla 5. El valor medio para el factor organización escolar es de 53.2 siendo 72 la puntuación máxima posible. El análisis de varianza muestra que existen diferencias en función del género y tener experiencia en cargos directivos o de gestión. Las mujeres puntúan significativamente más alto en este factor $(p<.05)$. Los docentes que han desarrollado o desarrollan un cargo directivo obtienen valores significativamente más altos que los docentes que nunca han tenido estas responsabilidad $(p<.05)$. Para el factor gestión de aula (ver Tabla 5) el valor medio es de 34.7 siendo 44 la máxima puntuación posible. El análisis de varianza muestra la existencia de diferencias significativas en función del género, la etapa educativa, los años de tutoría y el número de alumnos. Concretamente, las mujeres muestran mejores resultados en gestión de aula $(p<.001)$; los docentes de infantil y primaria son los que tienen mejores resultados $(p<.001)$; a mayor número de años como tutor/a mejor gestión de aula $(p<.001)$; los docentes que dan clase a menos de 50 alumnos obtienen mejores resultados $(p<.001)$. También se observan diferencias significativas para los años de ejercicio profesional, mostrando mejores resultados aquellos docentes con más de 20 años de experiencia $(p<.05)$.

Relación entre factores del contexto interno docente y $S Q T$

Los resultados del análisis de regresión jerárquica (ver Tabla 6), indican que el agotamiento emocional depende de la organización y gestión (10.9\%), la gestión de aula $(5.1 \%)$, una vez descontados los efectos de las variables socioprofesionales, de las cuales sólo los años de ejercicio profesional muestra un efecto significativo $(\mathrm{t}=3.92 ; p<.001)$. La despersonalización también viene explicada por la organización y gestión (5.3\%) y la gestión de aula (11.7\%), una vez desconta-

Tabla 3. Estadísticos descriptivos del MBI

\begin{tabular}{lccccrrrr}
\hline Escala y variable & $M(S D)$ & Rango & \multicolumn{2}{c}{ Riesgo bajo } & & \multicolumn{2}{c}{ Riesgo medio } & \multicolumn{2}{c}{ Riesgo alto } \\
\hline & & & $\mathrm{N}$ & $\%$ & $\mathrm{~N}$ & $\%$ & $\mathrm{~N}$ & $\%$ \\
\hline Agotamiento Emocional & $22.5(8.0)$ & $9-53$ & 691 & 50.8 & 476 & 35.0 & 193 & 14.2 \\
Despersonalización & $9.0(3.3)$ & $5-21$ & 372 & 27.3 & 853 & 62.7 & 135 \\
Realización Personal & $35.3(4.8)$ & $13-48$ & 680 & 50.0 & 543 & 39.9 & 137 & 10.1 \\
\hline
\end{tabular}


Tabla 4: Resultados MBI según variables sociodemográficas y profesionales

\begin{tabular}{|c|c|c|c|c|c|c|c|c|c|c|c|c|c|c|c|c|c|c|c|}
\hline \multicolumn{2}{|c|}{ Variable } & \multicolumn{6}{|c|}{ Agotamiento Emocional } & \multicolumn{6}{|c|}{ Despersonalización } & \multicolumn{6}{|c|}{ Realización Profesional } \\
\hline & & $M$ & $S D$ & $\mathrm{~F}$ & Bajo & Medio & Alto & $M$ & $S D$ & $\mathrm{~F}$ & Bajo & Medio & Alto & $M$ & SD & $\mathrm{F}$ & Bajo & Medio & Alto \\
\hline \multirow[t]{2}{*}{ Género } & Hombre & 22.2 & 7.5 & .580 & 49.0 & 40.7 & 10.2 & 9.9 & 3.5 & $49.4 * *$ & 18.3 & 67.4 & 14.3 & 35.4 & 4.9 & .066 & 50.5 & 39.0 & 10.5 \\
\hline & Mujer & 22.6 & 8.2 & & 51.4 & 32.5 & 16.1 & 8.6 & 3.1 & & 31.4 & 60.6 & 8.0 & 35.4 & 4.7 & & 50.0 & 40.1 & 9.9 \\
\hline \multirow[t]{3}{*}{ Edad } & $<=30$ & 20.4 & 6.4 & $7.35^{* *}$ & 60.3 & 33.8 & 6.0 & 8.7 & 3.0 & $2.32^{*}$ & 29.0 & 64.4 & 6.6 & 36.2 & 4.2 & $3.03 *$ & 57.1 & 36.0 & 6.9 \\
\hline & $31-50$ & 22.9 & 7.9 & & 48.8 & 35.5 & 15.7 & 9.1 & 3.3 & & 27.0 & 62.8 & 10.2 & 35.3 & 4.8 & & 49.5 & 40.4 & 10.0 \\
\hline & $>=51$ & 23.8 & 9.5 & & 44.7 & 35.0 & 20.3 & 9.8 & 3.5 & & 26.3 & 59.9 & 13.8 & 35.1 & 5.3 & & 41.5 & 43.8 & 14.7 \\
\hline \multirow{3}{*}{ Años prof. } & $<=10$ & 20.6 & 6.7 & $11.2^{* *}$ & 60.3 & 32.5 & 7.2 & 8.9 & 3.2 & 1.02 & 28.1 & 63.4 & 8.5 & 36.0 & 4.4 & $3.62^{*}$ & 55.1 & 38.1 & 6.8 \\
\hline & $11-20$ & 23.3 & 7.9 & & 45.7 & 36.5 & 17.9 & 9.1 & 3.3 & & 27.5 & 61.8 & 10.6 & 35.1 & 2.8 & & 47.3 & 40.3 & 12.3 \\
\hline & $>=20$ & 23.9 & 8.9 & & 43.5 & 36.8 & 19.8 & 9.4 & 3.3 & & 26.2 & 62.7 & 11.1 & 35.2 & 3.2 & & 45.9 & 42.0 & 12.1 \\
\hline \multirow[t]{3}{*}{ Etapa } & Inf./Prim & 22.1 & 8.0 & .980 & 52.7 & 31.9 & 15.4 & 8.5 & 3 & $13.1^{* *}$ & 33.7 & 59.7 & 6.6 & 35.8 & 4.6 & $4.47^{* *}$ & 54.2 & 37.1 & 8.7 \\
\hline & ESO & 22.5 & 7.8 & & 50.8 & 36.3 & 12.8 & 9.5 & 3.5 & & 21.8 & 64.7 & 13.4 & 35.2 & 4.8 & & 44.7 & 43.5 & 11.8 \\
\hline & $\mathrm{Bach} / \mathrm{CF}$ & 22.7 & 7.6 & & 44.4 & 42.0 & 13.5 & 9.5 & 2.8 & & 19.3 & 68.1 & 12.6 & 35.3 & 3.8 & & 48.3 & 41.1 & 10.6 \\
\hline \multirow[t]{2}{*}{ Cargo dir. } & $\mathrm{Si}$ & 22.5 & 7.3 & .092 & 48.3 & 38.9 & 12.8 & 9.0 & 3.4 & .191 & 28.9 & 60.9 & 10.2 & 35.8 & 4.7 & 3.02 & 54.0 & 36.9 & 9.0 \\
\hline & No & 22.2 & 8.2 & & 52.5 & 32.5 & 15.0 & 9.0 & 2.9 & & 26.5 & 63.8 & 9.7 & 35.3 & 4.7 & & 47.8 & 41.4 & 10.8 \\
\hline \multirow[t]{5}{*}{ Años tutor. } & 0 & 21.0 & 7.1 & $7.57^{* * *}$ & 58.4 & 33.5 & 8.1 & 9.1 & 3.1 & .137 & 25.4 & 65.6 & 9.1 & 35.2 & 4.8 & 1.03 & 45.5 & 42.6 & 12.0 \\
\hline & $<5$ & 21.2 & 7.3 & & 57.4 & 32.8 & 9.9 & 9 & 3.5 & & 26.4 & 63.8 & 9.9 & 35.7 & 4.5 & & 53.6 & 38.0 & 8.4 \\
\hline & $6-10$ & 22.7 & 8.0 & & 52.0 & 31.7 & 16.3 & 9.1 & 3.6 & & 31.0 & 57.9 & 11.1 & 35.5 & 4.8 & & 50.8 & 37.7 & 11.5 \\
\hline & $11-15$ & 23.8 & 8.0 & & 41.9 & 39.9 & 18.2 & 9 & 3.1 & & 28.4 & 61.5 & 10.1 & 35.8 & 3.9 & & 54.1 & 38.5 & 7.4 \\
\hline & $>15$ & 23.4 & 8.5 & & 44.1 & 37.9 & 17.9 & 9.1 & 3.2 & & 26.9 & 63.6 & 9.5 & 35.3 & 5.2 & & 47.9 & 41.5 & 10.5 \\
\hline \multirow[t]{5}{*}{$\mathrm{N}^{0}$ alumnos } & $<50$ & 21.5 & 7.6 & 2.5 & 53.6 & 31.4 & 15.0 & 8.0 & 2.9 & $5.72 * *$ & 38.3 & 56.0 & 5.7 & 36.1 & 4.6 & .528 & 54.0 & 38.3 & 7.6 \\
\hline & $50-100$ & 21.8 & 7.7 & & 50.5 & 38.0 & 11.4 & 9.5 & 3.5 & & 23.2 & 64.0 & 12.8 & 35.7 & 5.0 & & 52.2 & 35.7 & 12.1 \\
\hline & $101-150$ & 23.3 & 8.0 & & 46.3 & 37.7 & 16.0 & 9.6 & 3.4 & & 22.1 & 64.1 & 13.9 & 35.1 & 4.5 & & 47.0 & 42.0 & 11.0 \\
\hline & $151-200$ & 23.5 & 6.9 & & 45.7 & 39.5 & 14.8 & 9.4 & 3.0 & & 21.6 & 68.5 & 9.9 & 35.3 & 4.6 & & 46.3 & 42.0 & 11.7 \\
\hline & $>200$ & 22.2 & 8.3 & & 56.8 & 31.7 & 11.5 & 9.3 & 3.3 & & 19.7 & 71.6 & 8.7 & 34.9 & 4.8 & & 44.8 & 44.8 & 10.4 \\
\hline
\end{tabular}

Tabla 5: Resultados del ANOVA para factores del contexto docente

\begin{tabular}{|c|c|c|c|c|c|c|c|}
\hline \multicolumn{2}{|c|}{ Variable } & \multicolumn{3}{|c|}{ Organización y Gestión } & \multicolumn{3}{|c|}{ Gestión Aula } \\
\hline & & $M$ & $S D$ & $\mathrm{~F}$ & $M$ & $S D$ & $\mathrm{~F}$ \\
\hline Total & & 53.2 & 8.9 & & 34.7 & 4.6 & \\
\hline \multirow[t]{2}{*}{ Genero } & Hombre & 52.3 & 9.4 & $5.44 *$ & 33.8 & 4.4 & $25.05 * *$ \\
\hline & Mujer & 53.5 & 8.7 & & 35.1 & 4.9 & \\
\hline \multirow[t]{3}{*}{ Edad } & $<=30$ & 53.6 & 8.5 & 1.92 & 34.6 & 4.9 & .663 \\
\hline & $31-50$ & 52.8 & 9.0 & & 34.7 & 4.5 & \\
\hline & $>=51$ & 53.9 & 9.2 & & 35.0 & 4.6 & \\
\hline \multirow[t]{3}{*}{ Años prof. } & $<=10$ & 53.8 & 8.6 & 4.90 & 34.4 & 4.8 & $5.15^{*}$ \\
\hline & $11-20$ & 52.0 & 9.2 & & 34.6 & 4.5 & \\
\hline & $>=21$ & 53.4 & 9.0 & & 35.3 & 4.3 & \\
\hline \multirow[t]{3}{*}{ Etapa } & Inf/ Prim & 52.9 & 9.0 & 1.30 & 36.1 & 4.3 & $76.70 * *$ \\
\hline & ESO & 53.1 & 8.9 & & 33.0 & 4.3 & \\
\hline & $\mathrm{Bach} / \mathrm{CF}$ & 54.0 & 8.8 & & 34.0 & 4.6 & \\
\hline \multirow[t]{2}{*}{ Cargo dir. } & $\mathrm{Si}$ & 54.0 & 8.6 & $8.78^{*}$ & 35.0 & 4.3 & 3.72 \\
\hline & No & 52.5 & 9.0 & & 34.5 & 4.8 & \\
\hline \multirow[t]{5}{*}{ Años tutor. } & 0 & 53.5 & 9.2 & 1.14 & 32.9 & 4.8 & $15.26 * *$ \\
\hline & $<5$ & 53.8 & 8.7 & & 34.4 & 4.6 & \\
\hline & $6-10$ & 52.6 & 8.8 & & 34.9 & 4.4 & \\
\hline & $11-15$ & 52.2 & 9.4 & & 35.5 & 3.9 & \\
\hline & $>15$ & 53.2 & 8.9 & & 35.6 & 4.4 & \\
\hline \multirow[t]{5}{*}{$\mathbf{N}^{0}$ alumnos } & $<50$ & 53.7 & 8.7 & 1.90 & 36.7 & 4.5 & $31.25^{* *}$ \\
\hline & $50-100$ & 53.4 & 8.7 & & 34.1 & 4.3 & \\
\hline & $101-150$ & 53.3 & 9.2 & & 33.8 & 4.1 & \\
\hline & $151-200$ & 52.2 & 9.0 & & 33.3 & 4.4 & \\
\hline & $>200$ & 51.8 & 9.4 & & 33.8 & 4.7 & \\
\hline
\end{tabular}

$*=p<.05 * *=p<.001$ 
Tabla 6. Resultados del análisis de regresión jerárquica para las dimensiones del MBI

\begin{tabular}{|c|c|c|c|c|c|}
\hline & Modelo & $\mathrm{R} 2$ & $\Delta \mathrm{R} 2$ & $\beta$ & $\mathrm{F}$ \\
\hline \multirow[t]{3}{*}{ Agotamiento Emocional } & 1 Socioprofesionales & .046 & .046 & .207 & \multirow[t]{3}{*}{$36.32 * *$} \\
\hline & 2 Organización y gestión & .155 & .109 & -.283 & \\
\hline & 3 Gestión aula & .205 & .051 & -.246 & \\
\hline \multirow{3}{*}{ Despersonalización } & 1 Socioprofesionales & .064 & .064 & -.138 & \multirow[t]{3}{*}{$32.25^{* *}$} \\
\hline & 2 Organización y gestión & .053 & .117 & -.168 & \\
\hline & 3 Gestión aula & .079 & .196 & -.307 & \\
\hline \multirow[t]{3}{*}{ Realización Personal } & 1 Socioprofesionales & .027 & .027 & n.s. & \multirow[t]{3}{*}{$65.16^{* *}$} \\
\hline & 2 Organización y gestión & .060 & .086 & .135 & \\
\hline & 3 Gestión aula & .231 & .317 & .524 & \\
\hline
\end{tabular}

$* *=p<.001$

dos los efectos de las variables socioprofesionales, de las cuales sólo el género $(6.4 \%)$ muestra un efecto significativo $(\mathrm{t}=-4.19 ; p<.001)$. La realización personal viene explicada exclusivamente por la organización escolar $(8.6 \%)$ y principalmente por la gestión de aula $(23.1 \%)$.

\section{Discusión}

Este estudio tiene por objetivo determinar el nivel de prevalencia del burnout docente en centros concertados, así como describir las características pedagógicas y organizativas relacionadas que permiten explicar el SQT. A este respecto, se hipotetizaba que los docentes de centros concertados mostrarían un menor SQT que en otros estudios previos, y que la organización escolar y gestión de aula serían mejores predictores que las variables socioprofesionales.

La primera aportación de nuestro estudio ha sido describir la incidencia del SQT entre el profesorado de centros concertados. Los resultados muestran una incidencia menor a la obtenida en otros estudios realizados en España (Ayuso y Guillén, 2008; Durán et al., 2001; Manassero et al., 2000; Otero-López et al., 2009) o países de nuestro entorno (Marqués et al., 2005; Vercambre et al., 2009). Podría explicarse por la aplicación de un criterio restrictivo a la hora de considerar el nivel de riesgo, que acerca la prevalencia a los niveles encontrados por Extremera et al., (2010). En este sentido, consideramos que nuestros resultados son menos sesgados que en estudios previos y ello quizás pueda ser la causa por la que las diferencias entre docentes de centros públicos y concertados queden neutralizadas. En todo caso, podemos concluir que nuestra primera hipótesis no se ve confirmada y que, por tanto, no hay diferencias de SQT entre docentes de centros públicos y concertados.

$\mathrm{El}$ análisis de las combinaciones entre las dimensiones en riesgo del MBI de los 70 docentes de la muestra que se encuentran en situación de riesgo alto en dos dimensiones nos indica que la asociación más frecuente es agotamiento emocional y despersonalización $(44.3 \%)$, seguida de agotamiento emocional y baja realización personal (30\%) y de baja realización personal y despersonalización (25.7\%). Los resultados parecen ser coherentes con los obtenidos por algunos estudios, en los que el agotamiento emocional se identifica como la dimensión central del síndrome (Gil-Monte, 2007; Halbesleben y Demerouti, 2005; Melamed et al., 2006).

Desde el punto de vista de las variables socioprofesionales, parece haber ciertas diferencias de género en cómo se experimenta el SQT: las mujeres presentan mayor agotamiento emocional que los hombres, mientras que los hombres presentan mayor despersonalización. Estos resultados son consistentes con los de Ayuso y Guillen (2008) y Manassero et al. (2000). Según estos autores, estas diferencias se pueden explicar por cierta tendencia de los hombres a la impersonalidad, la insensibilidad o el distanciamiento ante los problemas que afectan a las personas, frente a una tendencia por parte de las mujeres al proteccionismo y/o la responsabilidad. Nuestros resultados también indican que la edad y los años de ejercicio profesional están relacionados con algunas dimensiones del síndrome, lo que coincide con la literatura existente (Marqués et al., 2005). Atendiendo a las etapas educativas en la que los docentes imparten docencia de forma preferente, no se han observado diferencias para el agotamiento profesional, pero sí en las otras dos dimensiones. Si como hemos dicho, se considerase el agotamiento emocional como la dimensión central del SQT, nuestros resultados inducen a pensar que la vivencia de exceso de demandas no varía sustancialmente con la edad de los alumnos, pero las diferencias en despersonalización y realización personal podrían indicar diferencias en el tipo de demandas y en los recursos de los docentes para cada etapa, tal como sugieren Otero-López et al. (2009). Algunos autores ya han incidido sobre una respuesta diferenciada ante las dificultades de conexión y/o la incertidumbre que 
supone trabajar con población adolescente, incluidos los problemas de conducta, la disminución del interés y motivación de los alumnos, el impacto del fracaso escolar acumulado y los conflictos derivados de la obligatoriedad de la escolarización en ciertas edades límite (Moriana y Herruzo, 2004). Otra explicación posible tendría que ver con el aumento de exigencia derivada de gestionar materias o programaciones más complejas (Vercambre et al., 2009).

Un segundo hallazgo de nuestro estudio ha sido describir las características pedagógicas y organizativas que se incluyen en el Contexto Interno Docente. Los dos factores que lo componen, organización escolar/ gestión pedagógica y gestión de aula, han permitido describir el estilo de gestión de los centros concertados. Resulta destacable que las mujeres muestren puntuaciones superiores en organización docente y gestión del aula. Nuestros resultados también permiten describir un perfil socioprofesional que contribuye a una buena gestión del aula: profesora de educación infantil o primaria, con años de experiencia docente y tutorial, con responsabilidad sobre grupos no muy numerosos.

En todo caso, y tal como se hipotetizaba, los factores del contexto interno docente son mejores predictores del SQT que las características socioprofesionales. Si bien se confirma el papel del género y los años de ejercicio profesional como variables socioprofesionales relevantes en el desarrollo del SQT, los resultados obtenidos prefiguran un patrón de relación entre el SQT y los factores del contexto docente según el cual: a) la organización y gestión deficiente de la escuela explica parte del agotamiento emocional (Lorente, Salanova, Martínez y Schaufeli, 2008) y b) la buena gestión de aula aparece como protectora ante la despersonalización e incrementa la satisfacción profesional.

En definitiva, consideramos que hemos encontrado argumentos sólidos que justifican la necesidad de superar la concepción del burnout docente como un fenómeno característico de profesores de mayor edad que imparten clases en la educación secundaria, para avanzar hacia un enfoque sistémico que considere el desarrollo profesional del docente y la escuela como modelo y cultura organizativa en la gestión y prevención del SQT. Desde este punto de vista, consideramos que el modelo pedagógico de la "escuela que aprende" es más saludable o de menor riesgo ante el SQT. Así pues, mejorar la organización escolar y la gestión pedagógica incide directamente en la reducción del agotamiento emocional. Además, dotar a los docentes de las competencias necesarias para una buena gestión del aula tendría un efecto preventivo, dada su incidencia positiva en evitar la despersonalización y aumentar la satisfacción personal. Estos resultados deberían tener implicaciones directas en los procesos de formación inicial y continua con docentes y directores/as de centros educativos.

Debemos considerar algunas limitaciones que acompañan nuestra investigación. Primero, el hecho de contar con una muestra compuesta exclusivamente por docentes de centros concertados catalanes limita la generalización de los resultados. Segundo, no es descartable que la organización interna de los distintos centros tenga relación con los resultados. Estudios posteriores en centros concertados de otras zonas de España o centros de carácter público ayudaría a generalizar los hallazgos de este estudio y verificar la baja prevalencia del SQT.

Para finalizar, la evidencia de capacidad predictiva de los dos factores del contexto interno docente sobre las dimensiones del MBI sugiere que su utilización para evaluar factores de riesgo en el SQT puede extenderse a nuevas investigaciones.

\section{Extended Summary}

Burnout syndrome usually affects people working in the so-called helping professions (health care, education, social services, etc.), whose working tasks have a core component of helping others and great emotional exchange. Burnout is described as a personal response to situations of professional maladjustment in front of job-related demands. It is considered as a phenomenon that includes: feelings of emotional exhaustion; attitudes of depersonalization (cynicism, detachment, coldness...) towards pupils, parents and peers, which is frequently associated to a decrease in teaching efficacy (Maslach, Schaufeli \& Leiter, 2001). In , according to Manassero et al. (2000), $40 \%$ of teachers have mild burnout symptoms, and between $3-4 \%$ have severe symptoms.

In the school context, literature identifies many variables related to burnout. For example, some of them are remarkable, such as: the teachers' commitment (Salanova, Schaufeli, Llorens, Peiró, \& Grau,
2000); classroom problems and disinterested pupils (Manassero et al., 1996); deficient working conditions (Peiró, 1992); school climate (Peiró \& Salvador, 1993); extreme working hours of teachers (Cladellas \& Castelló, 2011); or high demands on teachers (Vercambre, Brosselin, Gilbert, Nerrière, \& Kovess, 2009).

From the organizational point of view, authors coincide in considering burnout as the expression of maladjustment between the person and the organization, caused by the conflict or dissonance between the organization's and personal values (Salazar \& Beaton, 2000; Villanueva et al. 2008). Particularly, burnout is related to the breach of the worker's psychological contract with the organization, or to the discrepancy between the theoretical values or those reported by the organization, accepted when entering the organization, and those that are really lived daily (Leiter, 1999). In this sense, the model of learning school 
(Bolívar, 2001; Gairín, 2000; Santos Guerra, 2000) could be considered as the pattern of professional and organizational culture particular to healthier schools because: (a) it includes the management tendencies and postulates that characterize the best organizations (organizational strategy, relationship with the environment, innovation and assessment, management of knowledge, collaborative learning, etc.), and (b) responds to critical pedagogical approaches (shared educational project, pupil-oriented, joint construction of knowledge, etc.). These characteristics, at least theoretically, provide educational centres with a high capability to adapt to changes in their environment and to anticipate socio-educational demands that they have to meet.

Accordingly, this study aims to analyze the relationships between the teacher's inner context (classroom and school) and burnout, by trying to identify pedagogical and organizational characteristics that may help us prevent teacher burnout in accordance with healthier school and teaching models. Particularly, the hypotheses in our research are: (H1) classroom management with a pro-social style is a predictor of personal accomplishment; (H2) the organizational model defined as "learning school" is a predictor of personal accomplishment; and (H3) the factors of the teacher's inner context are better predictors of burnout than sociodemographic variables.

\section{Method}

\section{Participants}

A total of 1,360 Early Childhood, Primary and Secondary Education teachers from 38 state-assisted schools in took part in this study. The distribution of the sample was as follows: 15 centres and 532 teachers from the city of ; 9 centres and 372 teachers from cities with over 100,000 inhabitants; and 14 centres and 456 teachers from cities with less than 100,000 inhabitants. Inclusion criteria were to be working as a teacher in the selected educational centre in the academic year when data were collected.

\section{Instruments}

Questionnaire for sociodemographic and professional data: The questionnaire asks teachers about sociodemographic variables and other variables related to professional context and history: gender, age, years of teaching experience, educational stage, developing or having developed a managerial post, years of experience as a tutor, and number of pupils in their class.

Maslach Burnout Inventory (MBI): It allows us to measure the three dimensions of burnout: emotional exhaustion, depersonalization, and personal accomplishment, in its Spanish version. It consists of 22 items, divided into three subscales, which in our application have been used in terms of intensity (Calvete \& Villa, 1999).

Questionnaire of factors of the teacher's inner context: This questionnaire was specifically designed to carry out this research. 80 items were written down to collect information of: classroom pro-social leadership, management of teaching-learning processes, efficient achievement of objectives, teacher's participation, suitable distribution of functions and resources, existence of a sound and accepted educational project, good internal communication, team working, commitment to tutorial action and pupil guidance, articulation of syllabus through effective planning, orientation towards learning, and existence of internal assessment practices as a system to provide feedback for continuous improvement. A pilot study with 101 teachers from all educational levels led us to reduce the questionnaire to the 29-item version used in this study. The version used consists of two factors. A first factor called "School organization and educational management" consists of 18 items. The internal consistency of this factor is .89. The second factor, called "Classroom Management" consists of 11 items. The internal consistency of this factor is .84 .

\section{Procedure}

Centres were invited to participate in this research. Questionnaires were administered in teaching staff meetings, with a member of the research group attending. In compensation to their collaboration, every subject was given back their MBI corrected, and every centre the overall MBI results and the comparison with the total sample. Participating teachers were informed of data anonymity, and they expressed their consent to participate. To guarantee confidentiality, subjects were offered the possibility to refuse to participate in this study.

\section{Data analysis}

It has carried out a descriptive analysis of the three dimensions of MBI and internal contextual factors teachers. For dimensions of the MBI and teaching context factors have made successive one-factor ANOVAs for socio-professional variables. Finally, to test the extent to which the dimensions of the internal context predict teacher burnout, but controlling the effect of sociodemographic and professional, results from hierarchical regression analysis on the dimensions of the MBI in which socio-professional variables have been introduced first, the second term School Organization and finally the Hall Management. 


\section{Results}

According to the cut-off scores established to mark the MBI, $20.1 \%$ of subjects in our sample show high risk levels in only one dimension, $5.1 \%$ of the sample are at high risk in 2 dimensions, and $1.3 \%$ of subjects have high risk levels in the three MBI dimensions.

With regard to emotional exhaustion, the percentage of teachers at risk is significantly higher in women than in men $(p<.05)$. It also increases with age: concerning new teachers, twice as many teachers of between 31 and 50 years, and three times as many teachers older than 51 report emotional exhaustion $(p<.001)$. Similarly, among those teachers with more than 6 years of experience as tutors there is more emotional exhaustion than among those teachers not been tutors or have 5 or less years of experience as tutors $(p<.001)$. With regard to the dimension of depersonalization, men report significantly higher risk values than women $(p<.001)$. The educational stage where they teach is another variable with significant differences $(p<.001)$. In comparison with Primary, there are twice as many subjects at risk in Compulsory Secondary and Pre-University Education for this dimension, with Secondary Education being the stage with worse results. Finally, the number of pupils in class also presents significant differences, with those teachers with responsibility over less than 50 pupils being at a significantly lower risk of depersonalization $(p<.05)$. Results for personal accomplishment show that risk increases with age $(p<.05)$ and years of professional experience $(p<.05)$. Secondary Education teachers are those with lower personal accomplishment $(p<.001)$.

With regard to the teacher's inner context (School Organization/Pedagogical Management, and Classroom Management), women have significantly higher scores in Organization and Management $(p<.05)$. Teachers that have developed or are developing a managerial post also report significantly higher scores $(p<$ $.05)$. For Classroom Management, women present better results $(p<.001)$, as well as Early Childhood and Primary Education teachers $(p<.001)$; also the more years a teacher has been a tutor, the better their classroom management is $(p<.001)$, as well as those teachers with less than 50 pupils in class $(p<.001)$. There are also significant differences for the variable years of professional experience, with those teachers with more than 20 years of experience reporting better results $(p<.05)$.

The results of hierarchical regression analysis (see Table 6) indicate that emotional exhaustion depends on the organization and management $(10.9 \%)$, classroom management $(5.1 \%)$, after discounting the effects of variables professional groups, of which only years of practice showed a significant effect $(\mathrm{t}=3.92, p<.001)$. Depersonalization is also explained by the organization and management $(5.3 \%)$ and classroom manage- ment $(11.7 \%)$, after discounting the effects of socioprofessional variables, of which only gender $(6.4 \%)$ shows a significant $(\mathrm{t}=-4.19, p<.001)$. Personal fulfillment is explained solely by the school organization $(8.6 \%)$ and mainly for classroom management $(23.1 \%)$.

\section{Discussion}

This study aims to determine the prevalence of burnout level of teachers in aided schools, and to describe the pedagogical and organizational characteristics that explain related burnout. In this regard, it was hypothesized that teachers in aided schools show a lower burnout than in previous studies, and school organization and classroom management would be better predictors than socio-professional variables.

The first contribution of our study was to describe the incidence of burnout among teachers of aided schools. The results show a lower incidence than that obtained in other studies in Spain, but we believe that the results are less biased than in previous studies, and we can conclude that there are not differences in burnout among teachers of public and private schools.

Results appear consistent with those reported by some studies, in which emotional exhaustion is identified as the central dimension of the syndrome (GilMonte, 2007; Halbesleben \& Demerouti, 2005). From the point of view of socio-professional variables, seems to be some gender differences in how they experience burnout: women have greater emotional exhaustion than men, while men have greater depersonalization. Our results also indicate that age and years of practice are related to certain aspects of the syndrome, which is consistent with the literature (Marques et al., 2005). Following stages of education, no differences were observed for burnout, but in the other two dimensions. A possible explanation would be related to increased demand arising from managing complex materials or schedules (Vercambre et al., 2009).

A second finding of our study was to describe the pedagogical and organizational features that are included in the Domestic Context Teaching. Our results also allow to describe socio-professional profile that contributes to good classroom management: early childhood education teacher or parent, with years of teaching experience and tutorial, with responsibility for groups are not very numerous. As hypothesized, internal contextual factors are stronger predictors of teacher burnout that socio-professional characteristics: a) organization and management predicts emotional exhaustion (Lorente, Salanova, Martínez, \& Schaufeli, 2008), and b) good classroom management appears to be protective against the depersonalization and increase job satisfaction.

In short, we believe we have found strong arguments that justify the need to overcome the concept of teacher 
burnout as a phenomenon of older teachers who teach in secondary education, to move towards an approach that considers the professional development of teachers and school as a model and organizational culture in management and prevention of burnout. From this point of view, we believe that the pedagogical model of "learning school" is healthier or reduced risk of burnout. So, better school organization and educational management directly affects the reduction of emotional exhaustion. Consequently, providing teachers with the skills needed for good classroom management has a preventive effect, given its positive impact in avoiding the depersonalization and increased personal satisfaction

\section{Referencias}

Agencia Europea para la Seguridad y la Salud en el Trabajo (2009). Riesgos nuevos y emergentes para la seguridad y la salud en el trabajo. Recuperado de http://osha.europa. eu/es/publications/outlook/te8108475enc_osh_outlook

Ahghar, G. (2008). The role of school organizational climate in occupational stress among secondary school teachers in Tehran. International Journal of Occupational Medicine and Environmental Health, 21, 319-329.

Arís, N. (2009). El Síndrome de Burnout en los docentes. Electronic Journal of Research in Educational Psychology, 7, 829-848.

Ayuso, J. A. y Guillén, C. L. (2008). Burnout y mobbing en enseñanza secundaria. Revista Complutense de Educación, 19, 157-173.

Boada, J., de Diego, R. y Agulló, E. (2004). El burnout y las manifestaciones psicosomáticas como consecuentes del clima organizacional y de la motivación laboral. Psicothema, 16, 125-131.

Bolívar, A. (2001). ¿Dónde situar los esfuerzos de mejora?: Política educativa, centros o aula. Organización y Gestión Educativa, 39, 10-16.

Botella, L L., Longás, J. y Gómez, A. (2007). La construcción social del burnout en la profesión docente. Aloma, 21, 271-296.

Calvete, E. y Villa, A. (1999). Estrés y burnout docente: influencia de variables cognitivas. Revista de Educación, 319, 291-303.

Cladellas, R. y Badia, M. (2010). La gestión del tiempo de los profesores universitarios en función de la modalidad educativa: sus efectos psicosociales. Revista Española de Pedagogía, 246, 297-310.

Cladellas, R. y Castelló, A. (2011). Percepción del estado de salud y estrés, de profesorado universitario, en relación con la franja horaria de docencia. Electronic Journal of Research in Educational Psychology, 9, 217-240.

Durán, M. A., Extremera, N. y Rey, L. (2001). Burnout en profesionales de la enseñanza: Un estudio en educación primaria, secundaria y superior. Revisa de Psicología del Trabajo y de las Organizaciones, 17, 45-62.

Durán, M. A., Extremera, N., Montalbán, M. y Rey, L. (2005). Engagement y burnout en el ámbito docente:
Análisis de sus relaciones con la satisfacción laboral y vital en una muestra de profesores. Revista de Psicología del Trabajo y de las Organizaciones, 21, 145-158.

Extremera, N. Durán, M. A. y Rey, L. (2010). Recursos personales, síndrome de estar quemado por trabajo y sintomatología asociada al estrés en docentes de enseñanza primaria y secundaria. Ansiedad y Estrés, 16(1), 47-60.

Figueiredo-Ferraz, H., Gil-Monte, P. R. y Grau, E. (2009). Prevalencia del síndrome de quemarse por el trabajo (Burnout) en una muestra de maestros portugueses. Aletheia, 29, 6-15.

Gairín, J. (1999). La organización escolar: contexto y texto de actuación. Madrid: La Muralla.

Gairín, J. (2000). Cambio de cultura y organizaciones que aprenden. Educar, 27, 3185.

Gascón, S., Olmedo, M. y Ciccotelli, H. (2003). La prevención del burnout en las organizaciones: el chequeo preventivo de Leiter y Maslach. Revista de Psicopatología Clínica, 8, 55-66.

Gil-Monte, P. (2003). Burnout Syndrome: ¿Síndrome de quemarse por el trabajo, desgaste profesional, estrés laboral o enfermedad de Tomás? Revista de Psicología del Trabajo y de las Organizaciones, 19, 181-197.

Gil-Monte, P. (2005). El síndrome de quemarse por el trabajo ("burnout"). Una enfermedad laboral en la sociedad del bienestar. Madrid: Pirámide.

Gil-Monte, P. (2007). El síndrome de quemarse por el trabajo (burnout): Una perspectiva histórica. En P. Gil-Monte y B. Moreno (Eds.), El síndrome de quemarse por el trabajo (burnout). Grupos profesionales de riesgo (pp. 2141). Madrid: Pirámide.

Gil-Monte, P., Carlotto, M. S. y Gonçalves, S. (2011). Prevalence of burnout in a sample of Brazilian teachers. European Journal of Psychiatry, 25, 205-212.

Gil-Monte, P. y Peiró, J. M. (1999). Validez factorial del Maslach Burnout Inventory en una muestra multiocupacional. Psicothema, 11, 679-689.

Halbesleben, J. R. B. y Demerouti, E. (2005). The construct validity of an alternative measure of burnout: Investigating the English translation of the Oldenburg Burnout Inventory. Work and Stress, 19, 208-220.

Jennings, P. A. y Greenberg, M. P. (2008). The Prosocial Classroom: Teacher social and emotional competence in relation to student and classroom outcomes. Review of Educational Research, 79, 491-525.

Leiter, M. (1999). Burnout among teachers as a crisis in psychological contracts. En R. Vandenberghe y A. M. Huberman: Understanding and Preventing Teacher Bur nout (pp. 202-210). USA: Cambridge University Press.

Lorente, L., Salanova, M., Martínez, I. y Schaufeli, W. B. (2008). Extension of the Job Demands-Resources model in the prediction of burnout and engagement among teachers over time. Psicothema, 20, 354-360.

Llorens, S., García, M. y Salanova, M. (2005). Burnout como consecuencia de una crisis de eficacia: un estudio longitudinal en profesores de secundaria. Revista de Psicología del Trabajo y de las Organizaciones, 21, 55-70. 
Manassero, M. A., Fornés, J., Fernández, M. C., Vázquez, A. y Ferrer, M. V. (1996). Burnout en la enseñanza: Análisis de su incidencia y factores determinantes. Revista de Educación, 306, 45-67.

Manassero, M. A., García, E., Vázquez, A., Ferrer, V. A., Ramis, C. y Gili, M. (2000). Análisis causal del burnout en la enseñanza. Revista de Psicología del Trabajo y de las Organizaciones, 16, 173-195.

Mansilla, F. (2008). Manual de Riesgos Psicosociales en el trabajo: Teoría y Práctica. Recuperado de http:// www.psicologia-online.com/ebooks/riesgos/index.shtml

Marqués, A., Lima, M. L. y Lópes, A. (2005). Fuentes de estrés, burnout y estrategias de coping en profesores portugueses. Revista de Psicología del Trabajo y de las Organizaciones, 21, 125-143.

Martínez, I., Grau, R., Llorens, S., Cifré, E. y GarcíaRenedo, M. (2005). Efectos del desajuste obstáculos-facilitadores organizacionales en el estrés docente: Un estudio longitudinal. Revista de Orientación Educacional, 19, 59-78.

Maslach, C. (1999). Progress in understanding teacher burnout. En R. Vandenberghe \& A. M. Huberman (Eds.), Understanding and preventing teacher burnout: A sourcebook of international research and practice (pp. 221-222). New York: Cambridge University Press.

Maslach, C., Schaufeli, W. B. y Leiter, M. O. (2001). Job burnout. Anual Review of Psychology, 52, 397-422.

Maslach, C. y Jackson, S. E. (1986). Maslach Burnout Inventory. Manual research Edition. Palo Alto: University of California.

Melamed, S., Shirom, A., Toker, S., Berliner, S., y Shapira, (2006). Burnout and risk of cardiovascular disease: evidence, possible causal paths and promising research directions. Psychological Bulletin, 132, 327-353.

Moriana, J. A. y Herruzo, J. (2004). Estrés y burnout en pro- fesores. International Journal of Clinical and Health Psychology, 4, 597-621.

Otero-López, J. M., Castro, C., Villardefrancos, E. y Santiago, M. J. (2009). Job dissatisfaction and burnout in secondary school teachers: Student's disruptive behaviour and conflict management examined. European Journal of Education and Psychology, 2, 99-111.

Peiró, J. M. (1992). Desencadenantes estrés laboral. Madrid: Eudema.

Peiró, J. M. y Salvador, A. (1993). Control del estrés laboral. Madrid: Eudema.

Pillay, H. K., Goddard, R. y Wilss, L. A. (2005). Well-being, burnout and competence: Implications for teachers. Australian Journal of Teacher Education, 30, 22-33.

Salanova, M., Schaufeli, W., Llorens, S., Peiró, J. M. y Grau, R. (2000). Desde el "Burnout" al "Engagement": ¿Una nueva perspectiva?. Revista de Psicología del Trabajo y de las Organizaciones, 16, 117-134.

Santos Guerra, M. A. (2000). La escuela que aprende. Madrid: Morata.

Seisdedos, N. (1997). Manual MBI. Inventario "Burnout" de Maslach: Manual. Madrid: TEA.

Tardif, M. (2004). Los saberes del docente y su desarrollo profesional. Madrid: Narcea.

Unda, S., Sandoval, J. L. y Gil-Monte, P. R. (2008). Prevalencia del síndrome de quemarse por el trabajo (SQT) (burnout) en maestros mexicanos. Información Psicológica, 91/92, 53-63.

Vercambre, M., Brosselin, P., Gilbert, F., Nerrière, E. y Kovess, V. (2009). Individual and contextual covariates of burnout: a cross-sectional nationwide study of French teachers. Biomed Central Public Health, 9, 333.

Villanueva, R., Albadalejo, V. R., Ortega, P., Astasio, P., Calle, M. E. y Domínguez, V. (2008). Epidemiología del síndrome de bournout. Revista ROL de Enfermería, 31, 29-38.

Manuscrito Recibido: 11/06/2012

Revisión Recibida: 31/07/2012

Aceptado: 03/08/2012 